\section{Gas in the Portal Vein in Association with a Pelvic Abscess}

\section{Brit. med. F., 1967, 3, 288}

The radiological demonstration of gas in the portal vein was first reported by Wolfe and Evans (1955), and since then there have been nine further papers on this subject in the English language (Susman and Senturia, 1960 ; Sisk, 1961 ; Wiot and Felson, 1961 ; Barrett, 1962 ; Stewart, 1963 ; Guyer and Grainger, 1963 ; McCandless, 1964 ; Lazar, 1965 ; Fink and Boyden, 1966). The purpose of this communication is to record a further example of this apparently rare finding, which occurred in association with a pelvic abscess, and briefly to review the literature.

\section{CASE REPORT}

A 49-year-old woman was referred in 1964 with a rectovaginal fistula complicating radium therapy for carcinoma of the cervix in 1950. A loop sigmoid colostomy had been constructed in 1951 and was working satisfactorily, but the patient wished to be rid of it. Accordingly, it was decided to resect the fistula and the affected segment of rectum by an abdomino-anal pull-through excision. This was done in November 1964 under cover of the existing colostomy. Her postoperative course was complicated by pulmonary atelectasis and infection, which responded only slowly to physiotherapy and antibiotics, and delayed the closure of the sigmoid colostomy until 29 March 1965. After this procedure atelectasis recurred with infection and she was given a course of penicillin and streptomycin, which may well have masked the development of a pelvic abscess. However, she seemed to make good progress and was discharged home on the fourteenth postoperative day. She was readmitted three days later with abdominal distension and cardiovascular shock due to a bowel obstruction After resuscitation plain abdominal $x$-ray examination was carried out in the erect and supine positions, and showed the following features: (1) moderate small-bowel distension with fluid levels ; (2) a fluid level in the pelvis, apparently in a well-defined cavity, suggesting a high pelvic abscess; and (3) linear gas shadows in the liver extending to within $2 \mathrm{~cm}$. of the liver margin, as occurs when gas is present in the portal vein and its branches (see Fig.).

Laparotomy was performed on the day of admission and confirmed the diagnosis of a small-bowel obstruction, this being due to adhesions between the small bowel and the wall of a pelvic abscess closely related to the site of colostomy closure. The bowel

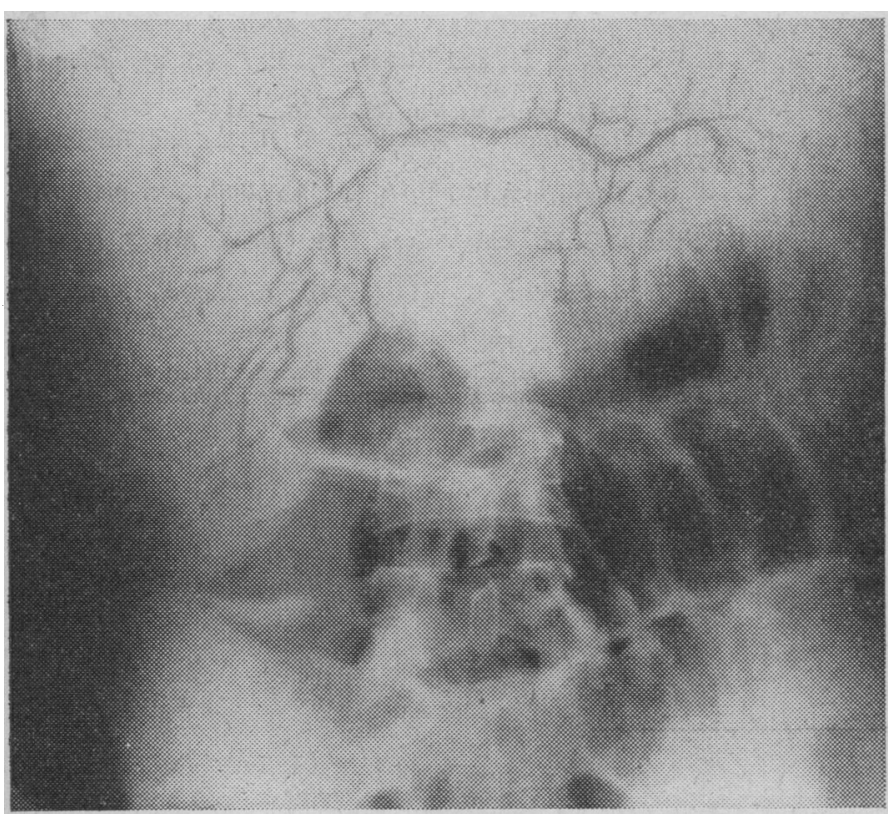

Supine abdominal $x$-ray plate of patient, showing moderate small-bowel distension and the portal veins outlined by gas. Note the gas shadows ap. roach to within $2 \mathrm{~cm}$. of the liver margin, a feature which serves to distinguish this, variety of intrahepatic gas from the more commonly occurring condition of gas in the bile-duct system. was only moderately distended and there was no evidence of ischaemia. The adhesions were freed and the abscess was drained. At the conclusion of the operation a tracheostomy was performed because of the previous episodes of postoperative pulmonary collapse and infection. Bacteriological culture of the pus obtained from the pelvic abscess gave a heavy growth of a coliform organism. During the operation the patient was given tetracycline, because, despite apparently adequate fluid and electrolyte replacement, her general condition deteriorated and it seemed likely that the abdominal condition was complicated by a Gram-negative septicaemia, though we were not able to prove this by positive blood culture. Her condition improved and she was subsequently treated in the intensive-care ward. Her initial progress was satisfactory and bowel function was re-established. Antibiotic therapy was changed from tetracycline to ampicillin on the basis of sensitivity tests. A pulmonary infarct was revealed radiologically on the seventh postoperative day, and this was complicated by a pyopneumothorax on the ninth day. Intercostal suction-tube drainage was instituted and chloramphenicol added to the antibiotic regimen, but she slowly deteriorated and died on the eighteenth postoperative day. Permission for necropsy was refused.

Throughout the postoperative period daily $x$-ray plates of her chest and abdomen were obtained, but in none of these was gas demonstrated within the hepatic shadow.

\section{COMMENT}

Gas in the portal vein is a rare finding, probably owing to its transient nature and to difficulty in obtaining good quality $\boldsymbol{x}$-ray films in severely ill patients. When obtainable, however, the radiological features are characteristic (see Fig.). An analysis of the pathology in 29 reported cases (see Table) emphasizes the combination of gross distension and impaired viability of the bowel. These features were noted in no fewer than 26 of the 29 cases. Susman and Senturia (1960) suggested three possible sources of gas in the portal vein: (1) gas within the distended bowel; (2) gas-forming organisms within the bowel wall; and (3) gas from an abscess cavity within the drainage area of the portal vein. In most reported cases the first two of these factors seem to be implicated in that the bowel was distended and ischaemic. Portal septicaemia may well be an accompanying feature, and in one reported case (Barrett, 1962) a positive blood culture for gas-gangrene organisms was obtained at operation from the portal vein.

\section{Pathological Lesions Present in 29 Recorded Cases of Gas in the Portal}

Vascular occlusions:

(a) Thrombosis or embolism of superior mesenteric (b) Strangulation of bowe

Necrotizing inflammations of intestine

Inflammation with less definite necrosis

Severe mediastinitis with septicaemia

Diabetic acidosis with bowel distension

Bowel distension was present in 27 patients, this distension often being described Bower diability or actual necrosis of the intestinal wall was present in 26 patients.

The present case is unique in that gas in the portal vein was associated with a proved intra-abdominal abscess containing gas, which may well have been forced into the portal venous system. However, even in this patient portal septicaemia has not been excluded as a contributory factor.

I am grateful to Professor J. C. Goligher for permission to publish this report and for his helpful advice in the preparation of the paper.

N. G. GrahaM,* M.B., F.R.A.C.S., F.R.C.S.

* From the Professorial Surgical Unit, the General Infirmary at Leeds.

\section{REFERENCES}

Barrett A F. (1962) Clin. Radiol, 13, 92

Fink, D. W. and Boyden, F. M. (1966). Radiology, 87, 741.

Guyer, P. B., and Grainger, K. (1963). Brit. 7. Radiol., 36, 379.

Lazar, H. P. (1965). Amer 7. dig. Dis., 10, 259.

McCandless, R G. (1964). Amer. F. Roentgenol., 92, 1162.

Sisk, P. B. (1961). Radiology, 77, 103.

Stewart, J. O. R. (1963). Brit. med. F., 1, 1328

Susman, N., and Senturia, H. R. (1960). Amer. . Roentgenol., 83, 847

Wiot, J. F., and Felson, B. (i96i). Ibid., 86, 920.

Wolfe, J. N., and Evans, W. A. (1955). Ibid., 74, 486. 